\title{
Aplicación del Análisis Probabilístico de Seguridad en el diseño de una planta productora de hidrógeno
}

\author{
Teresa Ruiz-Sánchez ${ }^{\mathrm{a}^{*}}$, Juan Luis Francois ${ }^{\mathrm{b}}$, Pamela F. Nelson ${ }^{\mathrm{b}}$ y M. Javier Cruz-Gómez \\ ${ }^{a}$ Instituto de Energías Renovables, UNAM, Privada Xochicalco, C.P. 62580, Temixco, Morelos. \\ ${ }^{b}$ Departamento de Sistemas Energéticos, Facultad de Ingeniería, UNAM, Paseo Cuauhnáhuac No. 8532, Col. Progreso, C.P. 62550, \\ Jiutepec, Morelos, México. \\ ${ }^{c}$ Facultad de Química, UNAM, Av. Universitaria No. 3000, Col. Copilco Universidad, Del. Coyoacán, C.P. 04360, México, D.F. \\ *truizsmx@yahoo.com.mx
}

Recibido 30 de Agosto de 2013, Aceptado 09 de octubre de 2013

\begin{abstract}
Resumen
Uno de los procesos que ha sido ampliamente investigado para la producción de hidrógeno, es el ciclo termoquímico Azufre-Yodo (S-I). Debido que, para esta tecnología, se utiliza un gran inventario de materiales tóxicos así como una alta temperatura en el proceso (obtenida por el acoplamiento de un reactor nuclear de alta temperatura) deben considerarse los sistemas de emergencia que se requieren para proteger las instalaciones, el ambiente y a la población. Dado el impacto que tendría una liberación accidental de los materiales del proceso y la cercanía con la planta nuclear es necesario que estos sistemas de emergencia sean lo más confiables posible. Así, los resultados del análisis de consecuencias son utilizados tanto, para la localización óptima de los sensores de gas que activan los sistemas de emergencia como, para la determinación de los flujos de las sustancias que se emplean para el control de la fuga. Por lo anterior, la metodología de Análisis Probabilístico de Seguridad (APS) y algunos estándares de la industria nuclear pueden ser aplicados a la instalación química para determinar las secuencias de falla que conllevan a estados finales de fuga no controlada. De esta manera, con base en los resultados obtenidos por el empleo de las técnicas de Análisis de Árboles de Eventos y Árboles de Fallas, se muestran los componentes más contribuyentes para la falla de dichos sistemas. Asimismo, en este trabajo se plantean algunas propuestas para incrementar la confiabilidad de los sistemas de emergencia. Adicionalmente, se presentan los resultados de las evaluaciones de estas propuestas, y su impacto en la probabilidad de las secuencias de fugas no controladas en una planta química que aún se encuentra la fase de diseño.
\end{abstract}

Palabras clave: Ciclo S-I, sistemas de emergencia, producción de hidrógeno, análisis probabilístico de seguridad, confiabilidad de sistemas.

\section{Introducción}

Desde finales del siglo pasado, una de las principales problemáticas mundiales a la cual nos enfrentamos es la energética. Los combustibles fósiles, que han sido hasta la actualidad la principal fuente de energía, presentan una perspectiva nada favorable para la continuidad de su uso, esto puede observarse en la relación de reservas y su duración con la creciente demanda, así como el impacto al medio ambiente al generar dióxido de carbono. Por ello, es necesario contar con nuevas formas para generar energía que sean eficientes pero sobre todo que contemplen los impactos ambientales en su proceso. Así, se prevé que el hidrógeno $\left(\mathrm{H}_{2}\right)$ sea el elemento que pueda cubrir esta necesidad.

Actualmente, uno de los procesos que, teóricamente, ha demostrado ser uno de los más eficientes para la producción de hidrógeno es el ciclo termoquímico Azufre-Yodo (S-I) acoplado a un reactor nuclear de muy alta temperatura (VHTR por sus siglas en inglés) [1]. El ciclo S-I consiste de las siguientes tres reacciones principales reportadas por General Atomic's [2]:

$2 \mathrm{H}_{2} \mathrm{O}+\mathrm{SO}_{2}+\mathrm{I}_{2} \rightarrow \mathrm{H}_{2} \mathrm{SO} 4+2 \mathrm{HI} \sim 120^{\circ} \mathrm{C}$

(Rxn Bunsen)
$\mathrm{H}_{2} \mathrm{SO}_{4} \rightarrow \mathrm{H}_{2} \mathrm{O}+\mathrm{SO}_{2}+1 / 2 \mathrm{O}_{2} \sim 830-900^{\circ} \mathrm{C}$

(Descomposición ácido sulfúrico, AS)

$$
2 \mathrm{HI} \rightarrow \mathrm{H}_{2}+\mathrm{I}_{2} \quad \sim 300-450^{\circ} \mathrm{C}
$$

(Descomposición ácido yodhírico, HIx’s)

Como puede observarse en las ecuaciones (1) a (3) la mayoría de las sustancias involucradas en el ciclo S-I son consideradas como peligrosas así es requerido un análisis de consecuencias para determinar los efectos que se tendría por la liberación de estas sustancias en los equipos e instalaciones, el ambiente y la población cercana. Para las simulaciones del análisis de consecuencias se utilizó el programa de cómputo Phast ver. 6.1 [3], el cual es ampliamente usado en la industria química.

Los resultados obtenidos en dicho análisis fueron presentados en un trabajo previo en esta revista [4] y un informe más extenso de éstos pueden obtenerse en la International Journal of Hydrogen Energy [5]. Así con base en los resultados de las simulaciones se definió la localización óptima de los sensores de gas. Asimismo, se 


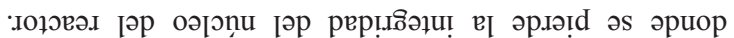

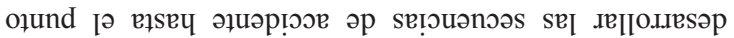

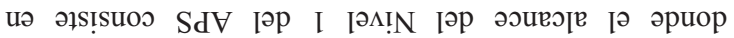

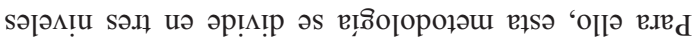

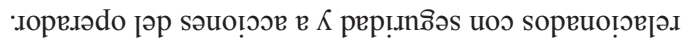

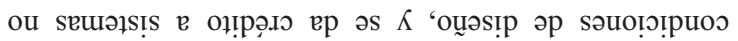

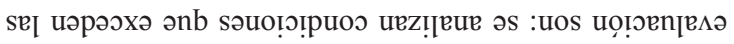

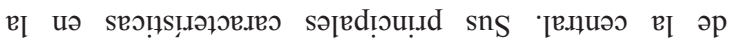

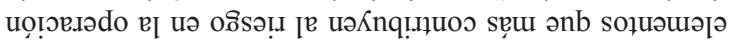

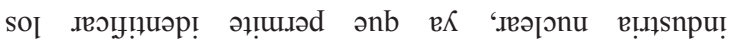

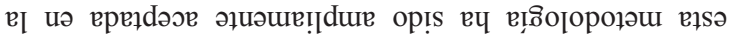

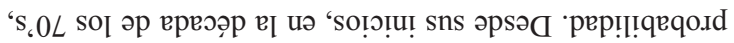

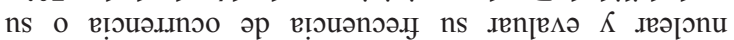

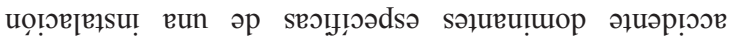
әр se!

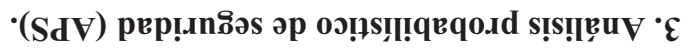

'sope.rny

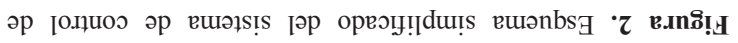

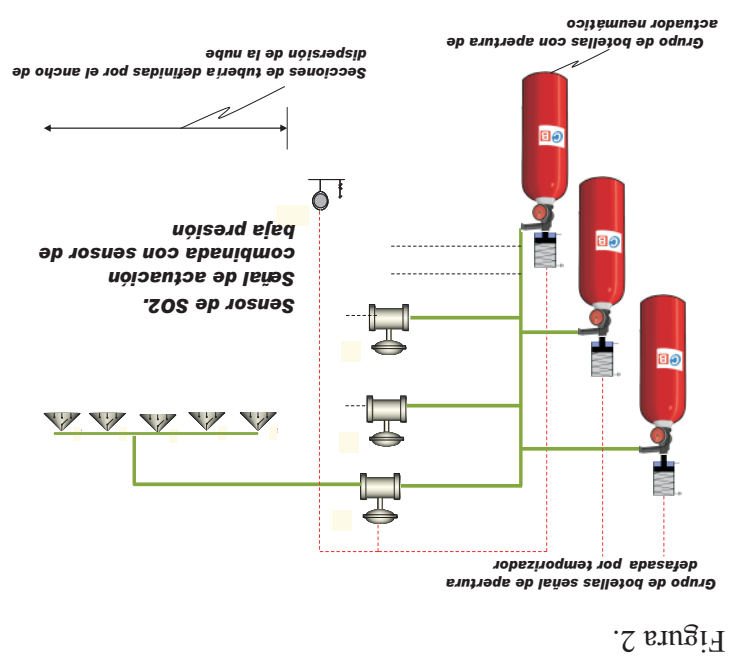

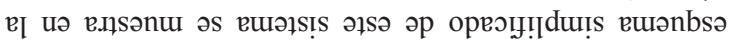

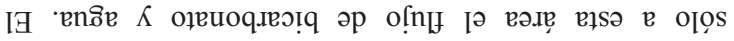

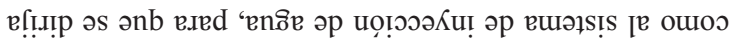

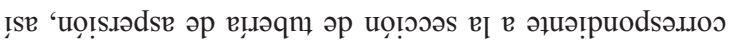

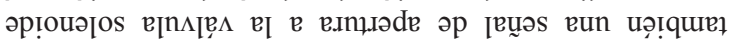

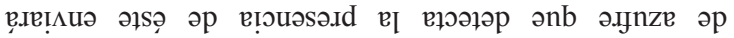

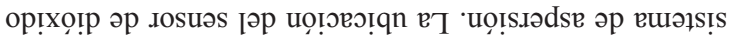

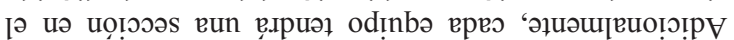

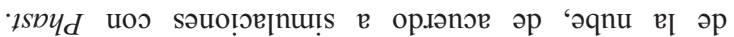

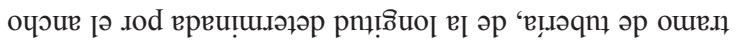

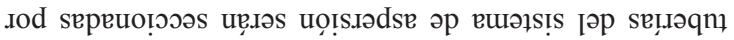

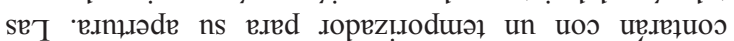

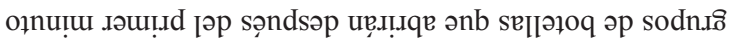

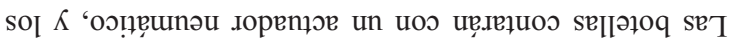

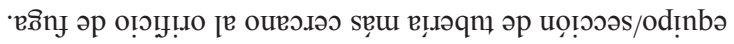

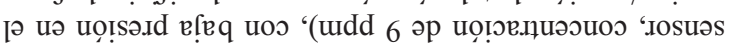

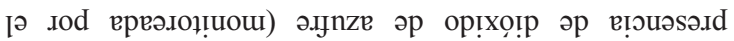

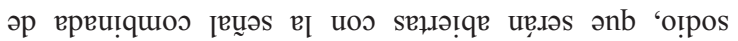

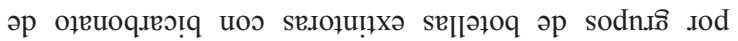

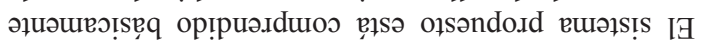

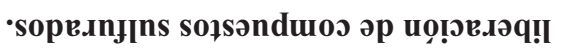

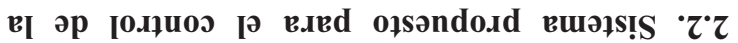

'opoК әр sołsənduo әр ио̣юв.əq!I

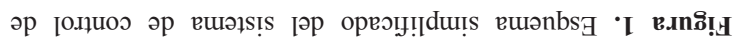

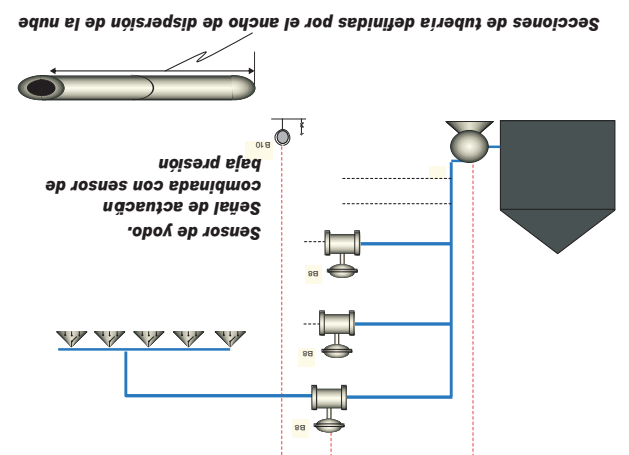

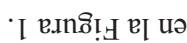

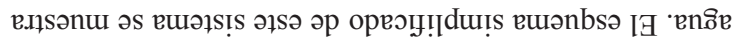

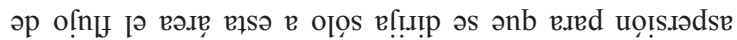

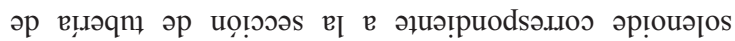

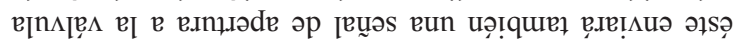

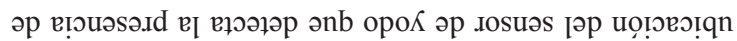

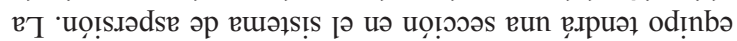

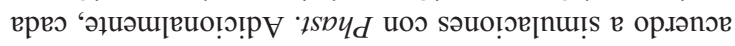

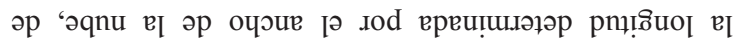

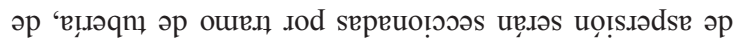

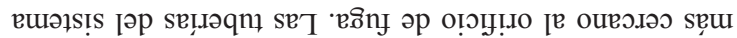

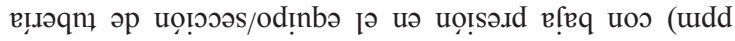

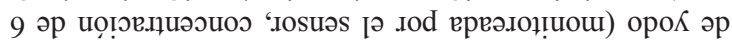

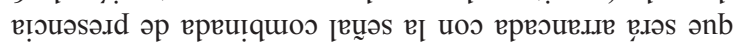

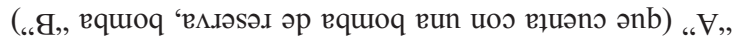

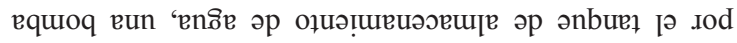

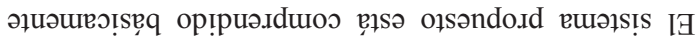

\section{-ороК әр sołsənduाoว әр ио̣эв.Jәq!I

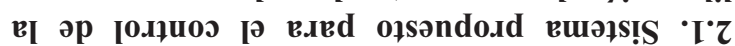

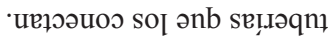

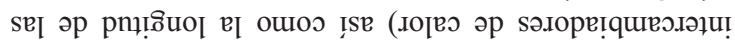

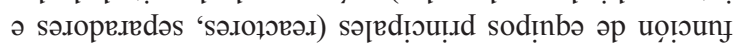

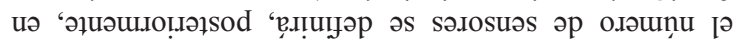

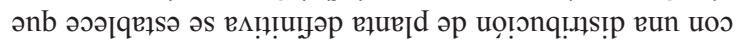

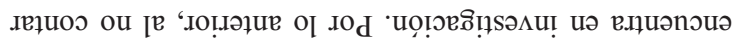
əs une osəวo.dd oKn

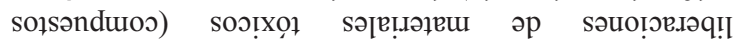

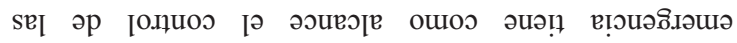

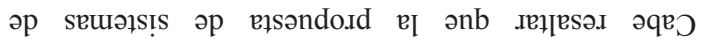

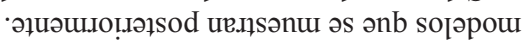

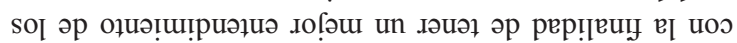

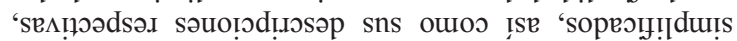

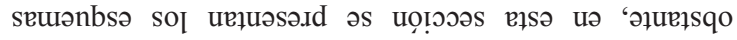

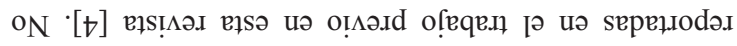

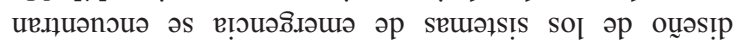

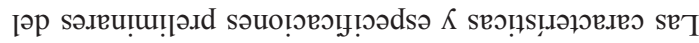

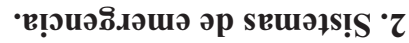

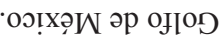

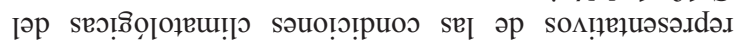

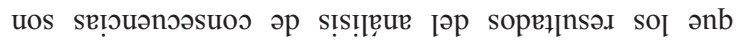

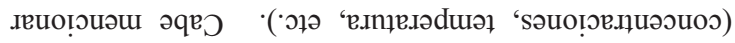

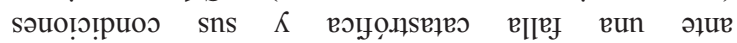

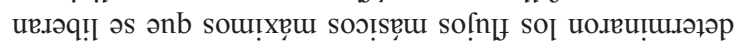


Mientras que los niveles 2 y 3 incluyen el proceso de degradación del núcleo, el comportamiento de la contención y los efectos externos de las posibles liberaciones radioactivas.

Como puede observarse en la Figura 3, la metodología de un Nivel 1 del APS se basa en dos tipos de diagramas lógicos:

- Árboles de Eventos: para determinar las posibles secuencias de accidente

- Árboles de Fallas: para obtener la probabilidad de fallas de sistemas

Una vez construidos los árboles de fallas se combinan y resuelven de manera conjunta con el árbol de eventos, obteniéndose las combinaciones de eventos básicos, llamados conjuntos mínimos de corte (CMC). Así, con las secuencias de accidente expresadas en términos de eventos básicos, es posible realizar la cuantificación probabilística. La reducción de árboles de fallas a CMC y la evaluación probabilística de cada secuencia de accidente se hace por medio de programas de cómputo. De esta forma pueden obtenerse los siguientes resultados:

- Secuencias de accidente dominantes. Sucesión de fallas y éxitos de sistemas/ componentes, así como acciones del operador que se presentan después del evento iniciador, y que tienen una contribución significativa a la probabilidad de fundición del núcleo.

- Eventos básicos más contribuyentes. Se obtiene la medida de cuánto contribuye un evento a la seguridad de la planta.

SISTEMAS DE MITIGACIÓN (para el control del accidente/evento iniciador)

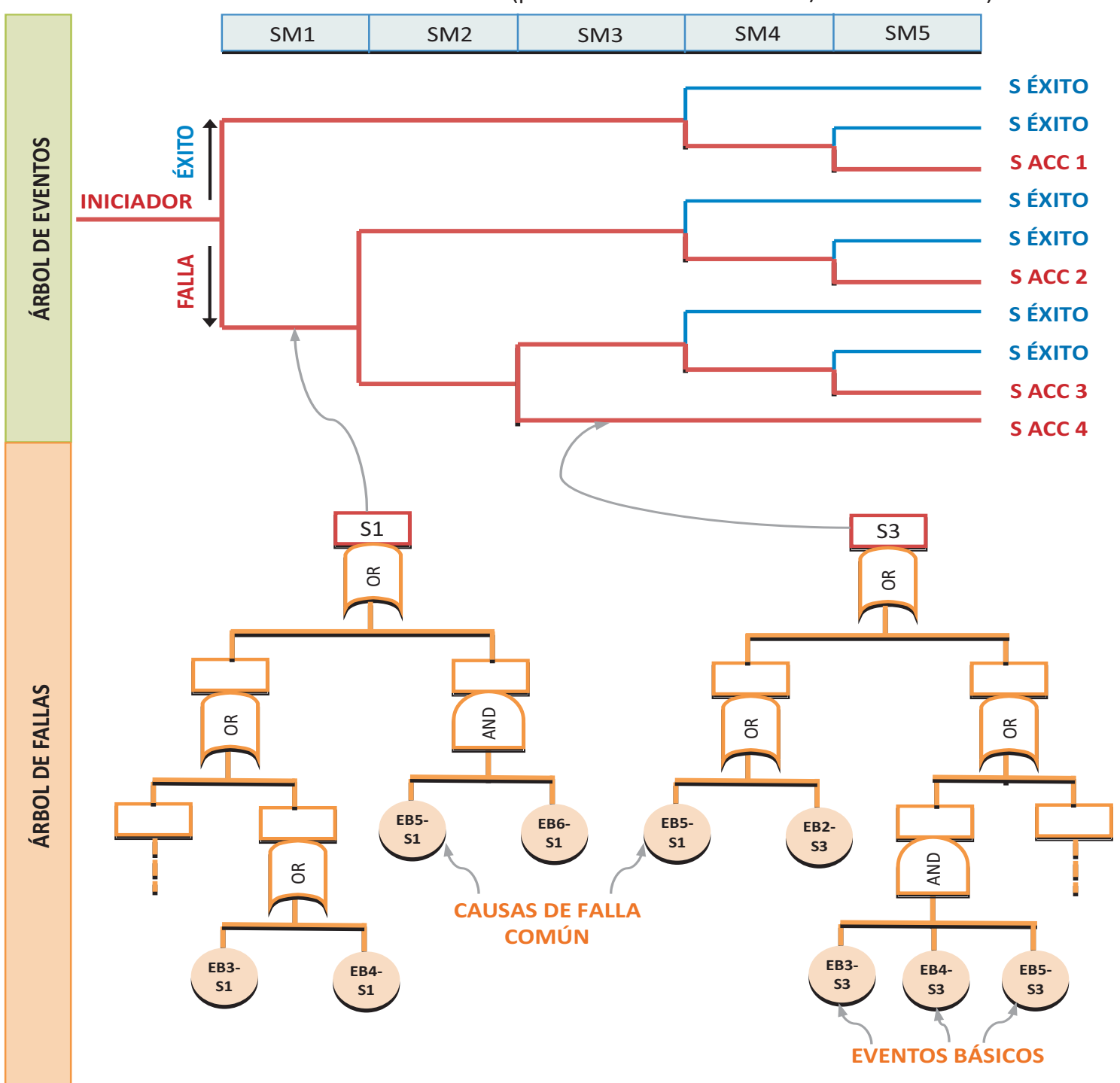

Figura 3. Representación esquemática de los modelos lógicos usados en el APS 


\subsection{Bases para el Desarrollo del APS de la Planta Productora de $\mathrm{H}_{2}$}

De acuerdo con la metodología de APS, inicialmente fueron desarrollados los árboles de eventos necesarios para representar la secuencia de accidente, tanto para la falla catastrófica como para la fuga probable, de acuerdo con la configuración de los sistemas propuestos (ver sección 2). Asimismo, posteriormente se desarrollaron los árboles de fallas requeridos para evaluar las fallas de los sistemas de mitigación representados en los árboles de eventos. Por ello, para la evaluación cuantitativa de los modelos, realizada en este trabajo usando el programa de cómputo Saphire ver. 6.7 [6], fue necesario asignar las probabilidades de falla de los eventos básicos.

Las tasas de falla de componentes para la asignación de las probabilidades de falla se obtuvieron de la referencia del American Institute of Chemical Engineers (AIChE) [7], ya que ésta es aplicable a la industria química. Este documento también se tomó como referencia para asignar la frecuencia de ocurrencia del evento iniciador "falla de la tubería", así para una fuga de $2.54 \mathrm{~cm}$ la frecuencia es de $5.7 \mathrm{E}-7 / \mathrm{h}$ (pag. 184) y para la falla catastrófica la frecuencia es de $2.68 \mathrm{E}-8 / \mathrm{h}$ (pag. 163). Adicionalmente, se incluyeron la indisponibilidad por mantenimiento tanto del tanque de almacenamiento como de la bomba de reserva.

Otras de las consideraciones para la asignación de probabilidades fueron:

- Período de calibración/pruebas de los sensores y temporizador: cada 6 meses

- Tiempo de mantenimiento de las bombas: cada 6 meses

- Tiempo de supervisión del tanque de almacenamiento de agua: cada año

- Tiempo para reparación del tanque de almacenamiento de agua: una semana

- Período de pruebas de la válvula de aire de instrumentos: cada año.

Así, se evaluaron las distintas secuencias de los árboles de eventos que tienen como estado final "fuga no controlada". En estos modelos se definieron el evento iniciador (en este caso ruptura de la tubería) y la secuencia de respuesta del equipo y acciones humanas en orden cronológico.

A continuación se detallan las consideraciones que se tomaron como base para el desarrollo de los modelos de los sistemas propuestos:

- Para el sistema de emergencia propuesto para el control de fugas de compuestos de yodo, la secuencia de las respuestas (sistemas de mitigación) quedó definida, por su temporalidad, de la siguiente forma (ver Figura 4):

- Actuación correcta de los sensores (presión y gas)

- Apertura de la válvula solenoide de la sección correspondiente

- Actuación del bombeo de agua

- Suministro (almacenamiento) de agua.
- Por otro lado, para el sistema de emergencia propuesto para compuestos sulfurados, los árboles de eventos desarrollados (ver Figura 6) tienen la misma secuencia que los modelos propuestos para los compuestos de yodo: primero la actuación de los sensores y luego los componentes agrupados en el sistema de aspersión de agua. Sin embargo, a este árbol se le adiciona la actuación del sistema de aspersión de bicarbonato (componentes: válvula solenoide de seccionamiento, temporizador y válvulas de actuación de las botellas extintoras).

\subsection{Evaluación de modelos del sistema de emergencia de compuestos de yodo.}

En la Figura 4 se muestra el árbol de eventos desarrollado para el control de la fuga de compuestos de yodo para cualquier caso de falla de tubería (catastrófica o probable). Asimismo, en la Figura 5 se muestra el árbol de fallas correspondiente, en el cual puede observarse que si se presenta la falla de cualquiera de los sensores (presión o gas), el estado final es de fuga no controlada.

\subsection{Evaluación de modelos del sistema de emergencia de compuestos sulfurados.}

En la Figura 6 se muestra el árbol de eventos desarrollado para el control de la fuga de compuestos sulfurados para ambos casos de falla. Asimismo, en las Figura 7 y 8 se muestran los árboles de fallas correspondientes a la falla de los sistemas de aspersión de agua y de bicarbonato, respectivamente. Cabe mencionar que la Figura 7 engloba los tres últimos sistemas de respuesta (mitigación) del árbol de fallas de la fuga de compuestos sulfurados.

Es importante recalcar que en el árbol de fallas del sistema de aspersión de agua (Figura 7) se incluye la indisponibilidad de las bombas por mantenimiento (IBOMB-A, I-BOMB-B), sin embargo, en la evaluación de los modelos el CMC que considera la indisponibilidad por mantenimiento de ambas bombas es eliminado. Lo anterior dado que, tanto en la industria nuclear como en la química, no está permitido que se realice mantenimiento a bombas que pertenecen al mismo sistema de manera simultánea.

Asimismo, el modelo de falla de los sensores es el mismo que se muestra en la Figura 5, la única diferencia es que para este sistema el sensor de gas que se utiliza es el de $\mathrm{SO}_{2}$.

En este árbol de eventos se observa que existen otros dos estados finales adicionales a los que se presentaron en el sistema de control de fuga de compuestos de yodo:

- Fuga con posible daño de equipo (corresponde a la secuencia donde se tuvo éxito sólo en la aspersión de agua) y

- Fuga con impacto ambiental (corresponde a la secuencia donde se tuvo éxito sólo en la aspersión de bicarbonato). 


\begin{tabular}{|c|c|c|c|c|c|c|c|}
\hline $\begin{array}{l}\text { FALLA } \\
\text { TUBERÍA }\end{array}$ & $\begin{array}{l}\text { ACTUACIÓN DE } \\
\text { SENSORES DE } \\
\text { GAS Y PRESIÓN }\end{array}$ & $\begin{array}{l}\text { APERTURA DE } \\
\text { VÁLVULA } \\
\text { SOLENOIDE }\end{array}$ & $\begin{array}{c}\text { ACTUACIÓN } \\
\text { BOMBA }\end{array}$ & $\begin{array}{l}\text { SUMINISTRO } \\
\text { DE AGUA }\end{array}$ & $\#$ & $\begin{array}{l}\text { NOMBRE DE LA } \\
\text { SECUENCIA }\end{array}$ & ESTADO FINAL \\
\hline \multirow[t]{6}{*}{ RUPT-TUB } & SENSOR & VALV-ABR & BOMBA-H2O & SUM-H2O & & & \\
\hline & & & & & 1 & OK & FUGA CONTROLADA \\
\hline & & & & & 2 & NO-SUM-H2O & FUGA NO CONTROLADA \\
\hline & & & & & 3 & NO-BOMBA & FUGA NO CONTROLADA \\
\hline & & & & & 4 & NO-VALV & FUGA NO CONTROLADA \\
\hline & & & & & 5 & NO-SENSOR & FUGA NO CONTROLADA \\
\hline
\end{tabular}

Figura 4. Árbol de eventos desarrollado para la evaluación de sistema de emergencia para el control de fugas de compuestos de yodo.

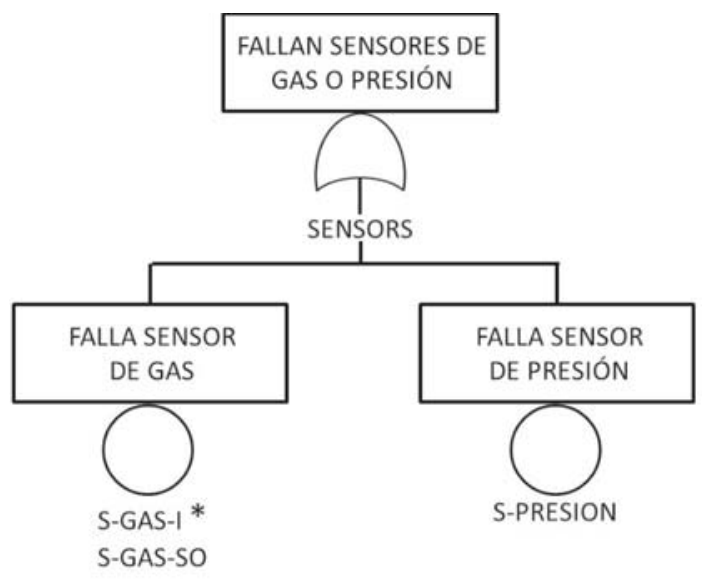

*S-GAS-I: Falla sensor de yodo para el sistema de emergencia para el control de liberaciones de compuestos de yodo o S-GASSO: Falla sensor de dióxido de azufre para el sistema de emergencia para el control de liberaciones de compuestos sulfurados

Figura 5. Modelo para la falla de los sensores de gas y presión
Se considera que el estado final de fuga, con posible daño, podría darse dado que el flujo de agua determinado para el sistema se calculó con un exceso para que se pueda absorber el calor de disolución del ácido sulfúrico; no obstante, se considera que podría provocarse alguna salpicadura al agregar agua al ácido sulfúrico mediante la aspersión. Así, existe la posibilidad de que algo de ácido tenga contacto con alguna tubería o equipo que pueda provocar la reacción del metal, produciendo su daño.

Asimismo, se tiene que el estado final de fuga, con impacto ambiental, puede presentarse dado que el flujo de bicarbonato está calculado con exceso, y se considera que se puede obtener la formación del ión bicarbonato, con la presencia del agua contenida en la humedad del ambiente, aunque sería menor la proporción del ión, por lo cual no se podría neutralizar toda la nube. Por lo anterior, ambos estados finales fueron evaluados como "fuga parcialmente controlada", como puede verse en la Figura 6.

\begin{tabular}{|c|c|c|c|c|c|c|}
\hline $\begin{array}{c}\text { FALLA } \\
\text { TUBERÍA }\end{array}$ & $\begin{array}{l}\text { ACTUACIÓN DE } \\
\text { SENSORES DE } \\
\text { GAS Y PRESIÓN }\end{array}$ & $\begin{array}{l}\text { SISTEMA DE } \\
\text { ASPERSIÓN DE } \\
\text { AGUA }\end{array}$ & $\begin{array}{l}\text { SISTEMA DE } \\
\text { ASPERSIÓN DE } \\
\text { BICARBONATO }\end{array}$ & $\#$ & NOMBRE DE LA SECUENCIA & ESTADO FINAL \\
\hline \multirow[t]{5}{*}{ RUPT-TUB } & SENSOR & S-H2O & $\mathrm{S}-\mathrm{NaHCO} 3$ & & & \\
\hline & & & & 1 & OK & FUGA NO CONTROLADA \\
\hline & & & & 2 & FUGA C/DAÑO EQUIPO & FUGA PARCIALMENTE CONTROLADA \\
\hline & & & & 3 & FUGA C/IMPACTO AMBIENTAL & FUGA PARCIALMENTE CONTROLADA \\
\hline & & & & 4 & NO-SISTEMAS & FUGA NO CONTROLADA \\
\hline & & & & 5 & NO-SENSOR & FUGA NO CONTROLADA \\
\hline
\end{tabular}

Figura 6. Árbol de eventos desarrollado para la evaluación de sistema de emergencia para el control de fugas de compuestos sulfurados. 


\section{Resultados.}

Los resultados de las evaluaciones de los modelos se muestran en la Tabla I. Aquí se muestran: la frecuencia de ocurrencia tanto para la falla catastrófica como para la fuga probable ( $2.54 \mathrm{~cm}$ de diámetro), dada la diferencia en el evento iniciador; la probabilidad condicional (dado que el evento iniciador ocurrió, es decir, se le asignó la probabilidad de uno), y los $\mathrm{CMC}$, el número total de combinaciones obtenidas y los cinco que tienen una mayor contribución, así como sus componentes. En esta tabla puede observarse que, como ya se mencionó anteriormente, sólo la evaluación de los modelos de fuga de compuestos sulfurados contiene secuencias de fuga parcialmente controlada.

En la Tabla I se observa que, para ambos sistemas, más del $85 \%$ de las veces que ocurra un evento de fuga puede tenerse como resultado estado final de fuga no controlada. Asimismo, el CMC más contribuyente es de orden uno, siendo el S-GAS su componente. Adicionalmente, se observa que el estado final de fuga no controlada se presenta una probabilidad condicional de $20.44 \%$.

Así, considerando estos resultados se hicieron las siguientes propuestas para mejora de los sistemas:

1. Una redundancia del sensor de gas y disminuir el tiempo de calibración de sensores de 6 a 3 meses.

2. Modificar la confiabilidad de los componentes a calidad nuclear utilizando las tasas de falla (medias) reportadas en el IAEA-TECDOC-508 [8]. Las tasas de falla remplazadas son:

- Bomba (tasa de falla a la demanda del AIChE 1.08E-2, IAEA 8.1E-03)

- Válvula solenoide (tasa de falla a la demanda del AIChE 2.83E-3, IAEA 1E-03)

- Temporizador (interruptor, tasa de falla del AIChE 49.6E-06/h, IAEA 8.6E-06/h)

\subsection{Evaluación de las propuestas de mejora.}

Los resultados de las evaluaciones de las propuestas de mejora se muestran en la Tabla II. Cabe mencionar que para esta evaluación sólo se muestran los CMC obtenidos con la propuesta 1 ya que éstos varían en la composición de los componentes.

Con estos resultados puede observarse que aplicando la propuesta de mejora 1 se reduce a la mitad la probabilidad de falla del sistema de emergencia, sin embargo, el CMC más contribuyente se vuelve de orden dos. Por otro lado, se aprecia que la probabilidad de ocurrencia de las secuencias de fuga no controlada, obtenida con la sustitución de equipo más confiable (calidad nuclear), es similar a la obtenida con la propuesta de colocar redundancia de los sensores de gas. Así, para determinar qué propuesta es más viable, se recomienda realizar un análisis de costos.

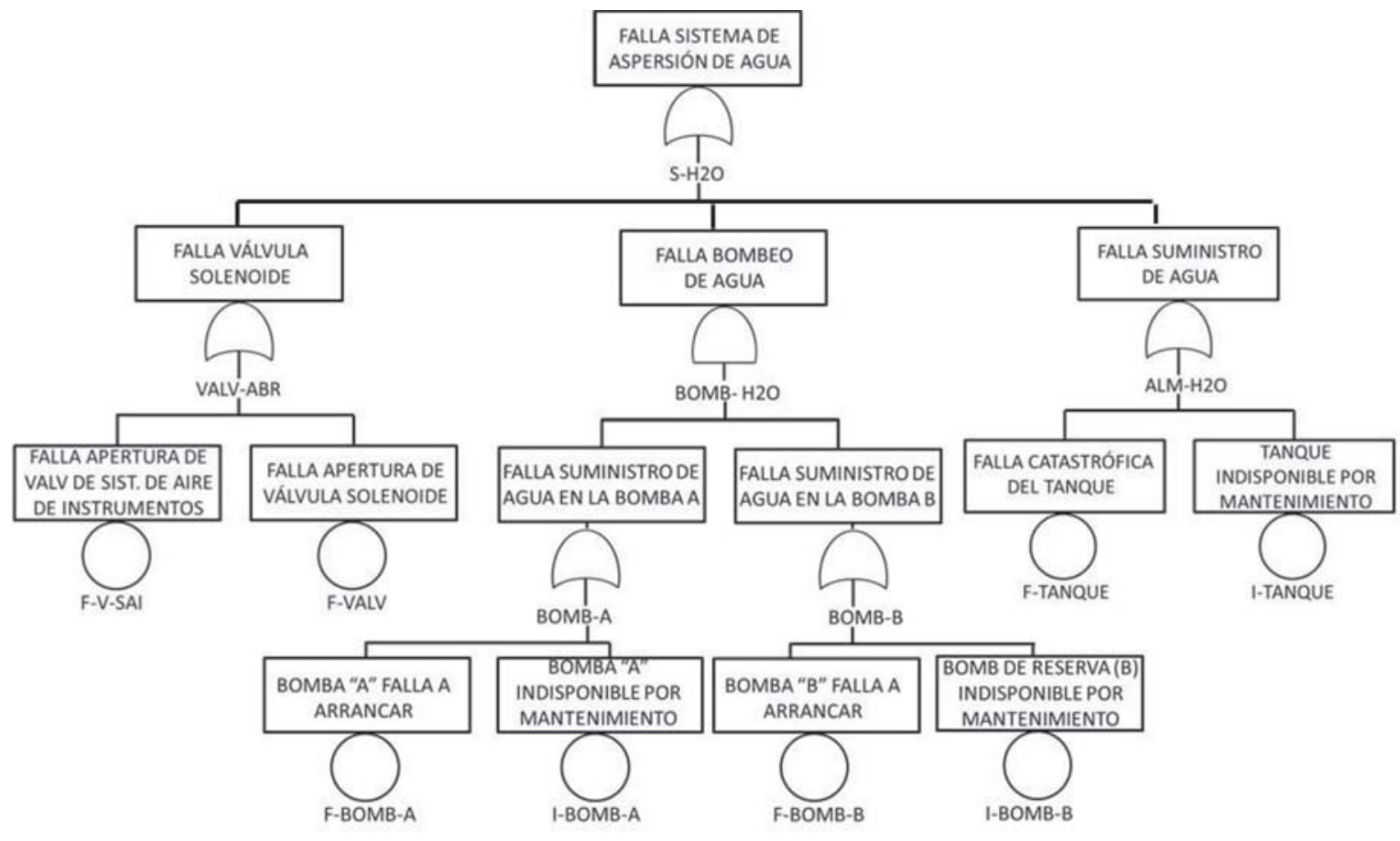

Figura 7. Modelo para la falla del sistema de aspersión de agua 


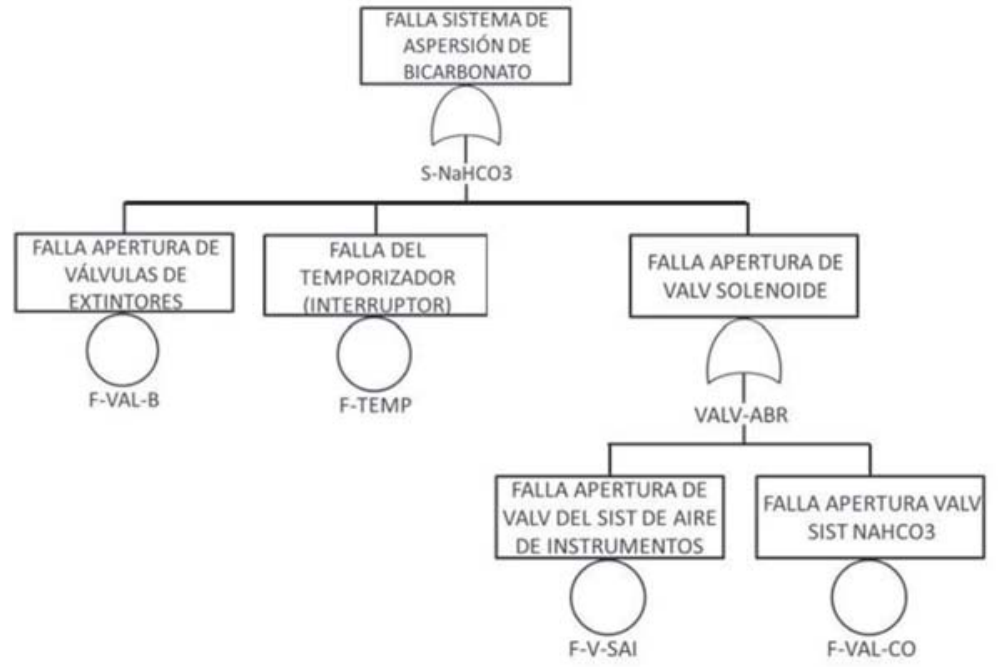

Figura 8. Modelo para la falla del sistema de aspersión de bicarbonato

Tabla 1. Resultados de la evaluación de los sistemas de emergencia (secuencias de fuga no controlada y parcialmente controlada)

\begin{tabular}{|c|c|c|c|c|}
\hline & \multicolumn{2}{|c|}{$\begin{array}{l}\text { Sistema para control de fugas compuestos } \\
\text { de yodo }\end{array}$} & \multicolumn{2}{|c|}{$\begin{array}{l}\text { Sistema para control de fugas compuestos } \\
\text { sulfurados }\end{array}$} \\
\hline & Falla catastrófica & Fuga probable & Falla catastrófica & Fuga probable \\
\hline $\begin{array}{c}\text { Frecuencia de ocurrencia -Fuga no } \\
\text { controlada }\end{array}$ & $\begin{array}{c}2.302 \mathrm{E}-08 / \mathrm{h} \\
(2.02 \mathrm{E}-04 / \mathrm{año})\end{array}$ & $\begin{array}{c}4.895 \mathrm{E}-07 / \mathrm{h} \\
(4.29 \mathrm{E}-03 / \mathrm{año})\end{array}$ & $\begin{array}{c}1.512 \mathrm{E}-08 / \mathrm{h} \\
(1.32 \mathrm{E}-04 / \mathrm{año})\end{array}$ & $\begin{array}{l}3.215 \mathrm{E}-07 / \mathrm{h} \\
(2.81 \mathrm{E}-03 / \text { año })\end{array}$ \\
\hline $\begin{array}{l}\text { Probabilidad condicional -Fuga no } \\
\text { controlada }\end{array}$ & \multicolumn{2}{|c|}{$8.588 \mathrm{E}-01$} & \multicolumn{2}{|c|}{$5.641 \mathrm{E}-01$} \\
\hline CMC (total) contribución/ componente(s) & \multicolumn{2}{|c|}{$\begin{array}{c}\text { CMC }(8) \\
97.04 \% \text { S-GAS } \\
1.83 \% \text { S-PRESION } \\
0.62 \% \text { I-TANQUE } \\
0.16 \% \text { F-VALV } \\
0.00 \% \text { F-TANQUE }\end{array}$} & \multicolumn{2}{|c|}{$\begin{array}{c}\text { CMC }(16) \\
91.04 \% \text { S-GAS } \\
3.61 \% \text { F-V-SAI } \\
2.23 \% \text { S-PRESION } \\
0.09 \% \text { I-TANQUE, S-TEMP } \\
0.06 \% \text { F-VALV, S-TEMP }\end{array}$} \\
\hline $\begin{array}{l}\text { Frecuencia de ocurrencia -Fuga } \\
\text { parcialmente controlada }\end{array}$ & & & $\begin{array}{c}5.478 \mathrm{E}-09 / \mathrm{h} \\
(4.80 \mathrm{E}-05 / \mathrm{año})\end{array}$ & $\begin{array}{c}1.165 \mathrm{E}-07 / \mathrm{h} \\
(1.02 \mathrm{E}-03 / \mathrm{año})\end{array}$ \\
\hline $\begin{array}{l}\text { Probabilidad condicional -Fuga } \\
\text { parcialmente controlada }\end{array}$ & & & \multicolumn{2}{|c|}{$2.044 \mathrm{E}-01$} \\
\hline CMC (total) contribución & & & \multicolumn{2}{|c|}{$\begin{array}{c}\text { CMC }(8) \\
96.19 \% \text { S-TEMP } \\
1.89 \% \text { I-TANQUE } \\
1.39 \% \text { F-VAL-CO } \\
1.39 \% \text { F-VALV } \\
0.06 \% \text { F-VALV-B }\end{array}$} \\
\hline
\end{tabular}

Tabla 2. Resultados de la evaluación de las propuestas de mejora a los sistemas de emergencia (secuencias de fuga no controlada y parcialmente controlada)

\begin{tabular}{|c|c|c|c|c|}
\hline & \multicolumn{2}{|c|}{$\begin{array}{l}\text { Sistema para control de fugas compuestos de } \\
\text { yodo }\end{array}$} & \multicolumn{2}{|c|}{$\begin{array}{c}\text { Sistema para control de fugas compuestos } \\
\text { sulfurados }\end{array}$} \\
\hline & $\begin{array}{c}\text { Propuesta de mejora } \\
1\end{array}$ & $\begin{array}{c}\text { Propuesta de mejora } \\
2\end{array}$ & $\begin{array}{c}\text { Propuesta de mejora } \\
1\end{array}$ & $\begin{array}{c}\text { Propuesta de mejora } \\
2\end{array}$ \\
\hline $\begin{array}{c}\text { Probabilidad condicional } \\
\text {-Fuga no } \\
\text { controlada }\end{array}$ & $4.602 \mathrm{E}-01$ & $4.588 \mathrm{E}-01$ & 29.2E-01 & $29.1 \mathrm{E}-01$ \\
\hline $\begin{array}{l}\text { CMC (total) } \\
\text { contribución/ } \\
\text { componente(s) } \\
\text {-Propuesta } 1\end{array}$ & \multicolumn{2}{|c|}{$\begin{array}{c}\text { CMC }(8) \\
97.07 \% \text { S-GAS, S-GASR* } \\
1.75 \% \text { S-PRESION } \\
1.0 \% \text { I-TANQUE } \\
0.26 \% \text { F-VALV }\end{array}$} & \multicolumn{2}{|c|}{$\begin{array}{c}\text { CMC }(16) \\
96.19 \% \text { S-GAS, S-GASR* } \\
1.01 \% \text { F-V-SAI } \\
0.83 \% \text { S-PRESION } \\
0.19 \% \text { I-TANQUE, S-TEMP }\end{array}$} \\
\hline
\end{tabular}




\section{Conclusiones}

Los sistemas de emergencia propuestos no incrementan el inventario de sustancias peligrosas incluidas en el proceso, debido a que se utiliza agua y bicarbonato de sodio. Para el inventario de estas nuevas sustancias debe considerarse el flujo másico liberado durante diez minutos en una falla catastrófica, por lo cual, cualquier liberación menor de las sustancias tóxicas estará completamente neutralizada mediante la actuación de los sistemas de emergencia. Asimismo, a pesar de que la liberación de compuestos sulfurados se presente a altas temperaturas, que provoquen la descomposición del bicarbonato, el carbonato que se produce es capaz de neutralizar a esta mezcla.

Las secuencias de fuga no controlada presentan una frecuencia de ocurrencia en el rango dentro de la frecuencia del evento iniciador (1.0E-03/año- $1.0 \mathrm{E}$ 04/año). Por ello, se evaluaron algunas propuestas de mejora, encontrándose que la incorporación de la redundancia de sensores de gas, y su reducción en el tiempo de calibración o el cambio de equipo a calidad nuclear, reduce a aproximadamente a la mitad la probabilidad de dichas secuencias. Otra de las modificaciones viables, y no evaluadas, es la recuperación de las funciones de mitigación mediante la incorporación de acciones humanas. Para ello, tendría que evaluarse la dispersión de la nube en función de la distancia y tiempo, y definir a qué funciones podría darse crédito.

Así, para la evaluación de la mejor propuesta, deberá hacerse un análisis de costo beneficio. Para ello, será necesario determinar el diseño final, tanto de los sistemas de emergencia como de proceso (y costo de la adición de las mejoras), así como definir los resultados del análisis de consecuencias como una función económica (costos de pérdidas de producción, de equipo y personas, así como multas por impacto ambiental).

Cabe recalcar que cualquier descontrol importante en la planta química (variaciones en temperatura, presión y flujo de proceso) enviará la señal de paro del reactor nuclear y viceversa.

\section{Agradecimientos}

Se hace un agradecimiento especial al Consejo Nacional de Ciencia y Tecnología (CONACYT) por la beca de doctorado otorgada a T. Ruiz-Sánchez, con la cual se hizo posible este trabajo. Adicionalmente, se agradece a la Universidad Nacional Autónoma de México por el apoyo brindado a través del proyecto de investigación PAPIIT IN106310.

\section{Referencias}

1. Brown, L. C.; Funk, J. F.; Pickard, P.; Marshall, A.; Showalter, S. K. High efficiency generation of hydrogen fuels using nuclear power - Final Techical Report, General Atomics Report GA-A24285, Rev. 01, December 2003.

2. Muradov, N. Z.; Veziroğlu; T. N. Int. J. Hydrogen Energy, 2008, 33, 6804-6839.

3. Phast 6.1 Professional, Process Hazard Analysis Software Tool, Norway: DNV, 1999.
4. Ruiz-Sánchez T.; Nelson, P. F. ; Francois, J. L., CruzGómez, M. J. REvista Qu\{imica Hoy, (Aplicación del Análisis de Consecuencias en la Propuesta de Sistemas de Emergencia para una Planta Productora de Hidrógenoartículo en proceso de publicación)

5. Ruiz-Sánchez T.; Nelson, P. F. ; Francois, J. L., CruzGómez, M. J. Int. J. Hydrogen Energy, 2012, 37, 6965 6975.

6. Idaho National Laboratory. https://saphire.inl.gov/ (accesado el 03 de marzo de 2013).

7. American Institute of Chemical Engineers (AIChE), Guidelines for Process Equipment Reliability Data with Data Tables. Center for Chemical Process Safety (CCPS), 1989, pp.127-212.

8. IAEA, Survey of Ranges of Component Reliability Data for Use In Probabilistic Safety Assessment, IAEA-TEDOC-508, Viena, 1989, pp 181-234. 